\title{
Reactivity of an Excess Electron with Monovalent Cations in Bulk Water by Mixed Quantum Classical Molecular Dynamics Simulations
}

\author{
RICCARDO SPEZIA a,b ${ }^{\mathrm{a}}$ CÉDRIC NICOLAS ${ }^{\mathrm{a}}$, FRANÇOIS-XAVIER COUDERT ${ }^{\mathrm{a}}$, PIERRE ARCHIREL ${ }^{\mathrm{a}}$, \\ RODOLPHE VUILLEUMIER ${ }^{\mathrm{a}, \mathrm{c}}$ and ANNE BOUTIN ${ }^{\mathrm{a}, *}$ \\ ${ }^{a}$ Laboratoire de Chimie Physique, UMR 8000, CNRS, Université de Paris-Sud, 91405 Orsay Cedex, France; ${ }^{\mathrm{b}}$ Dipartimento di Chimica, Università di \\ Roma, La Sapienza, Rome, Italy; ' Laboratoire de Physique Théorique des Liquides, UMR 7600, CNRS, Université Pierre et Marie Curie, 4 Place Jussieu, \\ 75252 Paris Cedex 05, France
}

(Received November 2003; In final form May 2004)

A mixed quantum classical molecular dynamics (QCMD) simulation of the silver and sodium cations in presence of an excess electron is reported. The silver cation is shown to be reduced by the hydrated electron and to form a stable, highly polarized, neutral atom. On the contrary, the sodium cation is not reduced and a metastable contact ion pair is observed. The resulting absorption spectra of both species are compared with experiments and shown to be in good agreement. Furthermore, the free energy curve for the charge separation was calculated and rationalized in terms of a thermodynamic cycle. Finally, a direct, reactive, molecular dynamics trajectory provides some useful informations on the reduction mechanism.

Keywords: Molecular dynamics; Silver cation; Absorption spectra; Thermodynamic cycle

\section{INTRODUCTION}

Understanding the elementary chemical events in terms of basic physical properties is a subject of considerable interest today. Among those, the electron transfer process has been widely studied, due to the importance of redox reactions in everyday physical chemistry and biophysics. The reduction of a metal ion was thoroughly investigated using pulse radiolysis techniques [1]. Several theoretical investigations have also been reported [2-4]. Even in the simplest case of metal atoms in polar solvents, the optical and electrical properties of the solvated atom, which strongly depend on the metal-solvent system, are not fully understood. In some cases, such as sodium in water, the ionization is complete and leads to two well-separated solvated species, i.e. the electron and the ion. In contrast, the reduction of silver cations in a radiolysis experiment or when a solvated silver salt is exposed to light leads to the formation of silver atoms, followed by a multistep coalescence into charged metallic clusters [5]. The role of the solvent is of critical importance as illustrated by the effect of the polarity of the solvent on the optical properties of solvated metal atoms [6]. The solvent indeed mediates effective interactions between the ion and the electron. These interactions depend on both the solvent and the ion, or atom properties.

The chemical nature of the cation is crucial for the equilibrium of the elementary redox processes in solution:

$$
e_{\mathrm{aq}}^{-}+M^{+} \leftrightharpoons M^{0}
$$

Silver cation solutions have been widely studied, owing to the existence of a monoelectronic reduction of the ion to the zerovalent state, to the stability of the bare cation in solution and to the easy complexation of the cation with various ligands. The silver aqueous solution was the first system studied by pulse radiolysis [7] and was recently revisited [5]. The optical absorption spectrum of transient single silver atoms has been observed in various solvents. This is followed by dimerization and multistep silver cluster formation. 
In contrast, the alkaline and earth-alkaline cations are not spontaneously reduced by an excess electron in solution, and only contact pairs are believed to be formed $[8,9]$.

Computer simulations are expected to contribute to the understanding of the difference in behavior between the two opposite cases: silver $\left(\mathrm{Ag}^{+}\right)$and sodium cation $\left(\mathrm{Na}^{+}\right)$in presence of an excess electron in bulk water. Here, we report a simulation study on these systems using mixed quantum classical molecular dynamics (QCMD). In the second section, the simulation technique is presented. The results of this study are reported in the third section and we finally conclude in the fourth section.

\section{MIXED QUANTUM CLASSICAL MOLECULAR DYNAMICS}

We performed a mixed QCMD of an excess electron in bulk water and also in the presence of a silver or sodium cation. The excess electron is treated quantum mechanically, using the Born-Oppenheimer approximation: a certain solvent/cation configuration $\mathbf{S}$ generates an effective electron-solvent/cation interaction potential $V_{\text {int }}(\vec{r}, \mathbf{S})$ where $\vec{r}$ denotes the electronic coordinates. The stationary Schrödinger equation for the excess charge in this effective potential is then solved at fixed solvent/cation coordinates:

$$
\left(T_{\mathrm{e}}+V_{\mathrm{int}}(\vec{r}, \mathbf{S})\right) \psi_{n}(\vec{r}, \mathbf{S})=E_{n}(\mathbf{S}) \psi_{n}(\vec{r}, \mathbf{S})
$$

where $T_{\mathrm{e}}$ denotes the electronic kinetic energy operator. The solution of the Schrödinger equation gives the electronic energy levels $E_{n}(\mathbf{S})$ and the corresponding wave function $\psi_{n}(\vec{r}, \mathbf{S})$, by diagonalization of Eq. (2) at each time step, i.e. for each solvent/cation configuration. Once the wave function is obtained, the quantum contribution to the forces acting on each classical particle is determined via the Hellmann-Feynman theorem [10,11] for each specific quantum state of the electron.

In the present work, the forces are always computed for the ground state of the excess electron $(n=0)$. The single-electron wave functions $\psi_{n}(\vec{r}, \mathbf{S})$ are expanded into a basis of $7 \times 7 \times 7$ spherical Gaussian functions centered on the nodes of a cubic lattice. The basis set parameters are identical to those used in Ref. [12] for simulation of a hydrated electron: the Gaussian exponent $\alpha$ is $0.357 \AA^{-2}$ and the basis grid extension is $10 \AA$, which corresponds roughly to half of the simulation box length. For the excess electron-water interaction we used the pseudo-potential developed by Turi and Borgis [13]. This pseudo-potential is based on a quantum $a b$ initio calculation for one water molecule plus an additional electron confined in a box in the static exchange theory limit. Details on the use of this pseudo-potential with a Gaussian grid basis set can be found in Ref. [12].

The electron/cation interaction was modeled using the one-electron semi-local pseudo-potential proposed by Durand and Barthelat [14]. The parameters were re-optimized in order to reproduce the properties of the isolated atoms using the Gaussian grid basis set used in the MD simulations. More details on the pseudo-potential parameters can be found in Ref. [15].

Water and cations interact via classical interaction potentials made of a coulombic and a 6-12 Lennard Jones (LJ) term. Water-water interactions are described by the SPC model [16], while $\mathrm{Na}^{+}$-water interaction by the Dang model [17]. For $\mathrm{Ag}^{+}-$water interaction there is no 6-12 LJ parameters in the literature, to our knowledge. We have thus adjusted silver-water LJ parameters in order to better reproduce structural and energetic properties of silver cation in water, performing purely classical MD runs of $\mathrm{Ag}^{+}$in $300 \mathrm{SPC}$ water molecules, with the same simulation conditions used for the QCMD simulation. We obtained $\epsilon_{\mathrm{Ag}^{+} / \mathrm{O}}=0.40 \mathrm{~kJ} / \mathrm{mol}$ and $\sigma_{\mathrm{Ag}^{+} / \mathrm{O}}=2.78 \AA$, with a point charge on silver for coulombic interactions, $q_{\mathrm{Ag}^{+}}=+1 e$ [15].

The simulations were carried out in a box of 300 and 800 SPC water molecules, a cation, $\mathrm{Ag}^{+}$or $\mathrm{Na}^{+}$, and an excess electron at ambient condition $\left(T=298 \mathrm{~K}\right.$ and $\left.\rho=1 \mathrm{~g} / \mathrm{cm}^{3}\right)$. Standard periodic boundary conditions were used with the Ewald summation technique to calculate the electrostatic interactions. The simulations were performed in the NVT ensemble using the Nosé-Hoover thermostat $[18,19]$ to ensure isothermal conditions. The integration of translation and rotation equation of motion was performed using the Gear predictorcorrector algorithm. The time step was fixed at $0.5 \mathrm{fs}$ and simulations were run typically during a few tens of picoseconds after a stabilization time. The Lanczos algorithm (ARPACK library) was used to solve the electron eigenvalue problem.

\section{RESULTS AND DISCUSSIONS}

\section{Absorption Spectra}

To calculate the electron absorption spectrum we used the cumulant expansion and the vibrationrotation decorrelation hypothesis [20], leading to the formula

$$
\begin{aligned}
I(E)= & E\left(1-e^{-\beta E}\right) \\
& \times \sum_{n=1}^{M}\left\langle\left|\mu_{0 n}\right|\right\rangle_{0} \exp \left[-\frac{\left(E-\left\langle E_{0 n}\right\rangle_{0}\right)^{2}}{\left\langle\delta E_{0 n}^{2}\right\rangle}\right]
\end{aligned}
$$


where $E_{0 n} \equiv E_{0 n}(\mathbf{S})$ is the solvent-dependent energy gap between the ground and the $n$th excited state, whereas $\mu_{0 n} \equiv \mu_{0 n}(\mathbf{S})$ is the corresponding transition dipole matrix. $\langle\ldots\rangle_{0}$ indicates an average over the solvent configurations with an electron equilibrated in its ground state. The vibration-rotation decorrelation approximation used in Eq. (3) assumes decorrelation between transition dipoles and transition energies: this approximation which may seem to be a drastic one, represents an excellent approximation to the absorption spectrum in the "slow modulation" (or "inhomogeneous broadening") limit $[15,21]$ and reduces the computational burden for a given signal/noise ratio.

The calculated and experimental spectra for the hydrated electron and in presence of a sodium or a silver cation are shown in Fig. 1. In all cases, the spectrum results from three sub-spectra corresponding to transitions from the s-type ground state to the three $p$-type excited states [12,15]. Within the model used, with no adjustment of the pseudopotential on the condensed phases properties, the overall agreement between experiments and simulations is fairly good for the three studied systems. However, the calculated spectrum for the hydrated electron does not reproduce the asymmetry of the experimental spectrum. Recent Car-Parrinello molecular simulations of the hydrated electron have been reported [22]. The optical absorption spectra calculated is more asymmetric in this case. The spatial distribution of the electronic charge of the excess electron often departs from an isotropic s-like shape and both localized lobes and solvation shells dynamically break and reform. This effect was not observed in our one electron mixed quantum classical simulation and we only observed a s-type electron in a water cavity. In the case of sodium and silver cations, however, our simulation model reproduces both the location and the width of
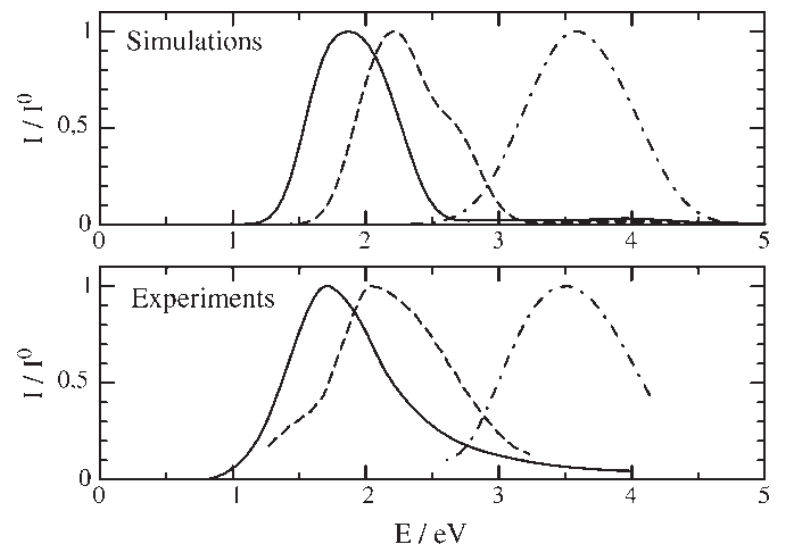

FIGURE 1 Absorption spectra of a hydrated electron (solid line), in presence of a sodium cation (dashed line) and in presence of a silver cation (dashed-dotted line). Simulation results (top panel) and experimental data (lower panel). the experimental spectra. A contact ion-electron pair is observed in the case of sodium [15] with an average electron-cation distance of $2 \AA$. Such a contact ion pair has also been observed recently by MD simulation of an electron in a reverse micelle [23]. In the case of silver, we observed the reduction of the cation. We found an average electron/silver distance of $\sim 0.5 \AA$. Although noticeably non-zero this value is well within the electron radius estimated from the width of the electron wavefunctions, i.e. $1.98 \AA$. The characterization of the neutral atom, i.e. an hydrophobic species in a polar solvent, was previously achieved by comparing our QCMD simulation with a Car-Parrinello study of the same system [24]. A large fluctuating induced atomic dipole of $\sim 2 \mathrm{D}$ on the neutral silver atom arose from both simulations, in very good agreement with the atomic dipolar excitonic state theory [25] that predicts for this system an atomic dipole of $\sim 1.96 \mathrm{D}$.

\section{Free Energy Curves}

Apart from the difference in nature of the reduced species, the two cations also differ by their ability to be reduced. In order to better characterize this property, we studied the free energy curves as a function of the electron/cation distance $r_{n}(\mathbf{S})$,

$$
r_{n}(\mathbf{S})=\left|\left\langle\Psi_{0}|\vec{r}| \Psi_{0}\right\rangle-\vec{R}_{\text {cat }}\right|=\xi
$$

For $\xi=0$, the electron is located on the atom. A contact electron/cation pair is formed as $\xi$ increases and, at large distances there are two distinct solvated species, the electron and the cation. We use the method proposed by Borgis and Staib to adapt the Umbrella Sampling technique in a system where a quantum particle is present $[15,26]$.

The free energy curves in the case of sodium and silver are shown in Fig. 2. The sodium curve reveals a minimum at $\sim 2.1 \AA$ (or $\sim 2.3 \AA$ ), and the silver one a minimum at $0.5 \AA$ (or $0.6 \AA$ ), for systems containing 300 or 800 water molecules, respectively. These values are similar to the average values found when performing standard (free) simulations. In the case of sodium the free energy strongly increases at short distances leading to difficulty in constraining the cation-electron distance to values smaller than $1.5 \AA$. The energy barrier to escape from the minimum is $\sim 0.08 \mathrm{eV}$ for sodium and $\sim 0.7 \mathrm{eV}$ in the case of silver. The sodium-electron pair appears to be metastable and a small perturbation is sufficient to separate the two species. The evolution of the energy levels of the excess electron are shown in Fig. 3 as a function of the cation-electron distance (it is found to be identical for systems with 300 and 800 water molecules). The general trend is a stabilization of the ground state when getting closer to the cation. The first three excited states are also stabilized but in 

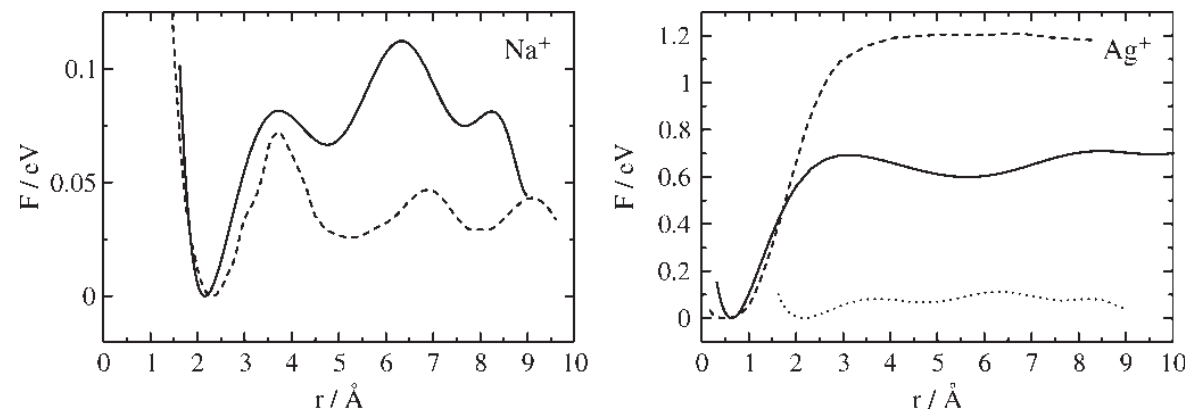

FIGURE 2 Free energy profiles as a function of electron-cation distance obtained for sodium (left panel) and silver (right panel) simulations. In each case, the solid line is obtained with 800 water molecules, and the dashed line with 300 water molecules. The sodium curve for 800 water molecules is also plotted on the right panel (dotted line).

a smaller quantity. In the case of sodium, the overall effect is small and the electron never gets closer than $2 \AA$ to the cation. The stabilization is much larger in the case of silver when the electron-cation distance gets lower than $2 \AA$. The three excited states are however much less stabilized than the ground state leading to the strong shift observed in the absorption spectrum compared to the hydrated electron case. The energy differences between the excited states and the ground state are plotted in Fig. 4, and compared to the energy differences in the simple case of the hydrated electron. The excess electron in presence of a cation tends to behave like the hydrated electron at large electron-cation distance as expected. The influence of the cation on the electron absorption spectrum is almost unnoticeable for distances greater than $9 \AA$.

\section{Thermodynamic Cycle}

This difference in behavior between sodium and silver can be rationalized in terms of a thermodynamic cycle. The free enthalpy, $\Delta G^{*}$, of the reduction reaction, Eq. (1), of the cation by the hydrated electron can be estimated by considering the solvation free enthalpies of the different species and the ionization potential in the gas phase. The thermodynamic cycle is presented in Fig. 5. For solvation free enthalpy of the hydrated electron, $\Delta G_{\text {solv }}\left(e^{-}\right)$, we have taken the value of $1.7 \mathrm{eV}$ [27].
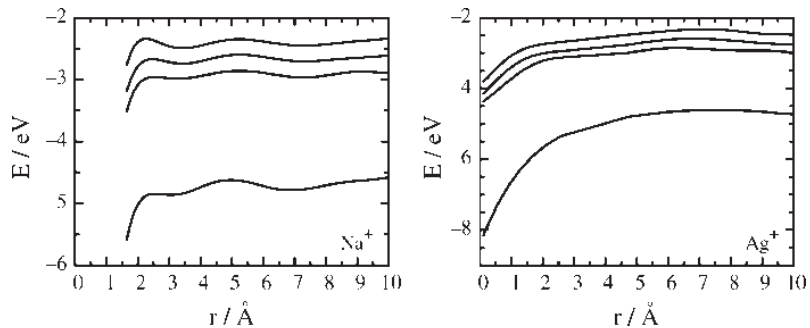

FIGURE 3 Evolution of the energies of the ground state and the three first excited states as a function of the electron-cation distance sodium (left panel) and silver (right panel). The plotted values correspond to an average along constraint molecular dynamics runs for a box with 800 water molecules.
The solvation free enthalpy of both neutral atoms are probably very small and were fixed to zero [28]. The solvation free enthalpy of the cation in water were estimated to -4.40 and $-5.12 \mathrm{eV}$ for sodium and silver, respectively, $[29,30]$. The gas phase ionization potentials of sodium and silver are 5.14 and $7.56 \mathrm{eV}$, respectively, [31].

Using the following equation:

$$
\begin{aligned}
\Delta G^{*}= & -\operatorname{IP}_{g}+\Delta G_{\text {solv }}\left(M^{0}\right)-\Delta G_{\text {solv }}\left(M^{+}\right) \\
& -\Delta G_{\text {solv }}\left(e^{-}\right)
\end{aligned}
$$

$\Delta G^{*}$ is thus estimated to be +0.96 and $-0.74 \mathrm{eV}$ for sodium and silver, respectively. Thermodynamics considerations show that the silver cation can be reduced by the hydrated electron in bulk water. On the other hand, as observed in our simulations, sodium can not be reduced to form the neutral metal atom.

\section{Direct Simulation of the Reduction Reaction}

From the above results, we can conclude that the silver atom should form spontaneously from the silver cation and the solvated electron. In order to check this point, we have performed direct simulations of the silver cation reduction reaction. Unconstrained simulations have been carried out, starting from 10 different configurations, for both 300
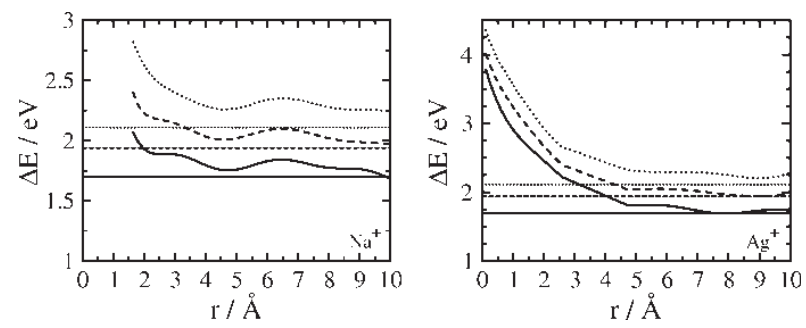

FIGURE 4 Evolution of the energy difference between three first excited states and the ground state as a function of the electroncation distance for sodium (left panel) and silver (right panel), with 800 water molecules. Full lines: $\left(E_{p 1}-E_{0}\right)$, dashed line: $\left(E_{p 2}-E_{0}\right)$ and dotted line: $\left(E_{p 3}-E_{0}\right)$. The horizontal lines correspond to the energies values obtained for the hydrated electron. 


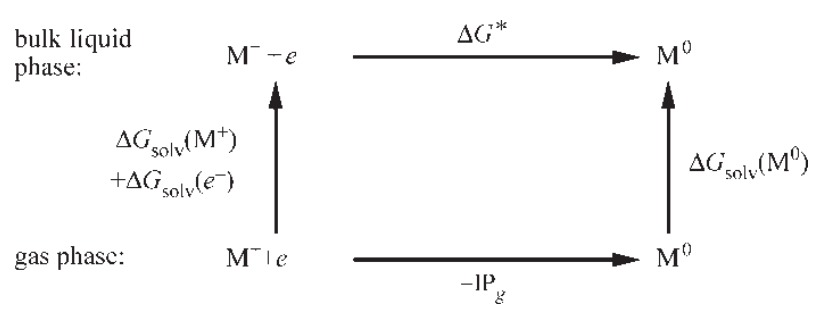

FIGURE 5 Thermodynamic cycle for the reduction of a cation in bulk liquid phase.

and 800 water boxes. The starting configurations correspond to a solvated electron in a solvent cavity separated from the cation by at least one water layer. At this stage, the electron properties are very similar to the free solvated electron ones (see Fig. 4). The evolution in time of the electron-cation distance along a selection of 7 different trajectories is presented in Fig. 6.

All but one trajectories spontaneously yield to the reduction of the silver cation in less than $10 \mathrm{ps,} \mathrm{for}$ both the 300 and 800 water molecules boxes. These trajectories reveal a general trend: during the first period the electron-cation distance is oscillating around an average distance equal or larger than $4 \AA$. It then suddenly drops to $1 \AA$ in $\sim 0.15$ ps. Then the electron-cation distance undergoes a rather slow decrease (from 1 to $0.5 \AA$ in about 0.8 ps) to the final excitonic state, oscillating around $0.5 \mathrm{~A}$. This second part of the reduction reaction is governed by the solvent reorganization. There is an important reorientation of water molecules in the first solvation shell between the contact ion-electron pair and the solvated silver atom. In the former, the water molecules are "hydrogen-bonded" to the electron [12], whereas the latter case is typical of a neutral atom solvation [24].

The activation energy of the reduction reaction can be estimated to $0.09 \mathrm{eV}(\sim 4 \mathrm{kT})$ from the free energy curve for 800 water molecules (Fig. 2). This value is

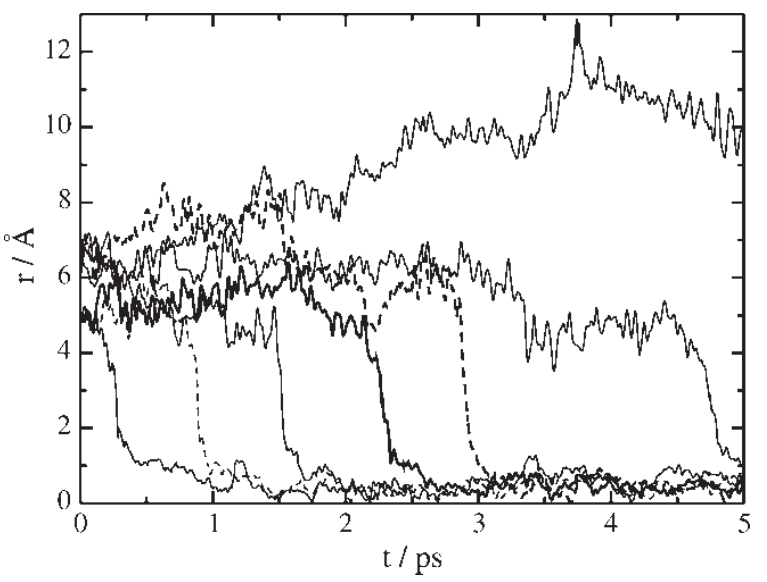

FIGURE 6 Evolution of the electron-silver cation distance versus simulation time for seven different initial configurations of systems with 300 and 800 water molecules. twice as small as the apparent activation energy of $0.18 \mathrm{eV}$ obtained experimentally by fitting the global rate constants on a wide range of temperature [5]. In the case of a 300 water molecules simulation, there is no apparent activation energy. We observe a surprising dependence of the free energy curve on the box size, which is presumably due to statistical uncertainties. Our results support the conclusion that the reaction between hydrated electrons and the silver ions is diffusion controlled, consistently with the evolution observed for the free trajectories.

\section{CONCLUSIONS}

The reactivity of an hydrated electron towards two types of cations have been studied in bulk water by mixed quantum classical simulation. We have found that the sodium cation is not reduced by an hydrated electron but forms an ion-electron contact pair. Free energy analysis reveals that this pair is metastable with respect to the fully separated state. On the other hand, the silver cation is reduced by an hydrated electron. The resulting neutral atom is very stable and is akin to a dipolar excitonic state. The absorption spectra have been calculated in all cases and a good agreement with experiments is observed. Constrained molecular dynamics have been performed in order to investigate the evolution of the electron energy levels and the total free energy of the system with mean electron-cation distances. The observed shift of absorption spectra in presence of a cation is due to a stabilization of the electron ground state with decreasing electroncation distance. For distances larger than $10 \AA$, no influence of the cation on the hydrated electron properties can be noticed. The spontaneous reduction reaction of the silver cation has also been observed by direct molecular dynamics simulation. It shows that a complete description of the reaction should include the solvent reorganization as the electron-cation distance is decreased. Such simple mixed quantum-classical molecular dynamics simulations are shown to reproduce the details of solvated species properties in bulk fluid and help in better understanding the chemistry of solvated atoms and cations. A study of the reduction process over a wide range of temperature is now in progress in order to increase our insights in the charge-transfer-to-solvent processes.

\section{Acknowledgements}

We wish to acknowledge B. Lévy, M. Mostafavi and A. H. Fuchs for fruitful discussions. We thank the Institut du Développement et des Ressources en Informatique Scientifique (IDRIS) of the CNRS for generous allocations of computer time and 
the European Union Marie Curie training site UDYCHEM for foundings and a grant for one of us (RS).

\section{References}

[1] Buxton, G.V., Greenstock, C.L., Helman, W.P. and Ross, A.B. (1988) "Critical review of rate constants for reactions hydrated electrons, hydrogen atoms and hydroxyl radicals in aqueous solution", J. Phys. Chem. Ref. Data 17, 513.

[2] Sprik, M., Impey, R.W. and Klein, M.L. (1986) "Electron-ion interactions and ionization in a polar solvent", Phys. Rev. Lett. 56, 2326.

[3] Borgis, D. and Staib, A. (1994) "Excited states of hydrated electron and aqueous chloride by computer simulations", Chem. Phys. Lett. 230, 405.

[4] Staib, A. and Borgis, D. (1996) "Reaction pathways in the photodetachment of an electron from aqueous chloride: a quantum molecular dynamics study", J. Chem. Phys. 104, 9027.

[5] Mostafavi, M., Lin, M., Wu, G., Katsumura, Y. and Muroya, Y. (2002) "Pulse radiolysis study of absorption spectra of $\mathrm{Ag}^{0}$ and $\mathrm{Ag}_{2}^{+}$in water from room temperature up to $380^{\circ} \mathrm{C}^{\prime \prime}$, J. Phys. Chem. A 106, 3123.

[6] Belloni, J., Mostafavi, M. and Amblard, J. (1994) In: Rossky, P.J. and Gauduel, Y., eds, Ultrafast reaction Dynamics and Solvent Effects (American Institute of Physics, Woodbury).

[7] Mulvaney, P. and Henglein, A. (1990) "Formation of unstabilized oligomeric silver clusters during the reduction of $\mathrm{Ag}^{+}$ions in aqueous solution", Chem. Phys. Lett. 168, 391.

[8] Gelabert, H. and Gauduel, Y. (1996) "Short-time electron transfer processes in ionic aqueous solution: counterion and H/D isotope effects on electron-atom pairs relaxation", J. Phys. Chem. 100, 13993.

[9] Renou, F., Mostafavi, M., Archirel, P., Bonazzola, L. and Pernot, P. (2003) "Solvated electron pairing with earth alkaline metals in THF. 1. Formation and structure of the pair with divalent Magnesium", J. Phys. Chem. A 107, 1506.

[10] Feynman, R.P. (1939) "Forces in molecules", Phys. Rev. 56, 340.

[11] Hellmann, J. (1937) Einführung in die Quantenchemie (Deuticke, Leipzig).

[12] Nicolas, C., Boutin, A., Lévy, B. and Borgis, D. (2003) "Molecular simulation of a hydrated electron at different thermodynamic state points", J. Chem. Phys. 118, 9689.

[13] Turi, L. and Borgis, D. (2002) "Analytical investigations of an electron-water molecule pseudo potential. II. Development of a new pair potential and molecular dynamics simulations", J. Chem. Phys. 117, 6186.

[14] Durand, P. and Barthelat, J.C. (1975) "A theoretical method to determine atomic pseudopo-tentials for electronic structure calculations of molecules and solids", Theor. Chim. Acta 38, 283.
[15] Spezia, R., Nicolas, C., Archirel, P. and Boutin, A. (2004) "Molecular dynamics simulations of the $\mathrm{Ag}^{+}$or $\mathrm{Na}^{+}$cation with an excess electron in bulk water", J. Chem. Phys. 120, 5261.

[16] Berendsen, H.J.C., Postma, J.P.M., van Gunsteren, W.F. and Hermans, J. (1981) Interaction models for water in relation to protein hydratation (Dordrecht, Reidel).

[17] Dang, L.X. (1995) "Mechanism and thermodynamics of ion selectivity in aqueous solutions of 18-Crown-Ether: a molecular dynamics study", J. Am. Chem. Soc. 117, 6954.

[18] Nosé, S. (1984) "A unified formulation of the constant temperature molecular dynamics methods", J. Chem. Phys. 81, 511.

[19] Hoover, W.G. (1985) "Canonical dynamics: equilibrium phase-space distributions", Phys. Rev. A 31, 1695.

[20] Tarjus, G. and Bratos, S. (1984) "Theory of infrared and raman spectra of diluted van der Waals solutions: simple product approximation and its extension", Phys. Rev. A 30, 1087.

[21] Staib, A. and Borgis, D. (1995) "Molecular dynamics simulation of an excess charge in water using mobile Gaussian orbitals", J. Chem. Phys. 103, 2642.

[22] Boero, M., Parrinello, M., Terakura, K., Ikeshoji, T. and Liew, C. (2003) "First-principles molecular-dynamics simulations of a hydrated electron in normal and supercritical water", Phys. Rev. Lett. 90, 226403.

[23] Laria, D. and Kapral, R. (2002) "Electron solvation in aqueous reverse micelles: equilibrium properties", J. Chem. Phys. 16, 7712.

[24] Spezia, R., Nicolas, C., Boutin, A. and Vuilleumier, R. (2003) "Molecular dynamics simulations of a silver atom in water: dipolar excitonic state evidence", Phys. Rev. Lett. 91, 208304.

[25] Logan, D.E. (1986) “Comment on electron-ion interactions and ionization in a polar solvent", Phys. Rev. Lett. 57, 782.

[26] Borgis, D. and Staib, A. (1996) "Quantum adiabatic umbrella sampling: the excited state free energy surface of an electronatom pair in solution", J. Chem. Phys. 104, 4776.

[27] Zhan, C.G. and Dixon, D. (2003) "The nature and absolute hydration free energy of the solvated electron in water", J. Phys. Chem. B 107, 4403.

[28] Dubois, V., Archirel, P. and Boutin, A. (2001) "Monte Carlo simulations of $\mathrm{Ag}^{+}$and $\mathrm{Ag}$ in aqueous solution. Redox potential of the $\mathrm{Ag}^{+} / \mathrm{Ag}$ couple", J. Phys. Chem. B 105, 9363.

[29] Burgess, J. (1978) Metal Ions in Solution (Ellis Horwood, Chichester, UK).

[30] Tissandier, M., Cowen, K., Feng, W., Gundlach, E., Cohen, M., Earhart, A., Jr., R, T. and Coe, J. (1998) “The proton's absolute aqueous enthalpy and Gibbs free energy of solvation from cluster ion solvation data", J. Phys. Chem. A 102, 9308.

[31] Parr, R.G. and Yang, W. (1989) Density-Functional Theory of Atoms and Molecules (Oxford University Press, New York). 